\title{
Niveles de Ansiedad Rasgo y Bienestar en jugadores de fútbol profesional de Chile durante la cuarentena por COVID-19 \\ Levels of Trait Anxiety and Well-being in Chilean professional soccer players during the COVID-19 quarantine
}

\author{
Paula Francisca Ortiz Marholz, Luis Marcel Valenzuela Contreras, Joel Barrera Diaz \\ Universidad Católica Silva Henríquez (Chile)
}

Resumen. En respuesta a la aparición del COVID 19 y la pandemia ocasionada, los países tomaron medidas para manejar las curvas de infección. Han obligado a permanecer en confinamiento y esta estrategia afectó a diversos contextos, dentro de los cuales, se encuentra el ámbito deportivo, suspendiendo competiciones, partidos oficiales, entrenamientos en cancha, transmisiones deportivas, etc. Sin embargo, en el caso de los profesionales del fútbol, se mantuvieron en preparación física a través de plataformas tecnológicas. El objetivo de este trabajo es explorar los niveles de ansiedad rasgo, de sensación de bienestar y la relación entre ambas variables en futbolistas profesionales de cuatro clubes de Chile con el fin de contribuir al diagnóstico sobre cómo la crisis de Covid-19 puede haber afectado a la realidad deportiva en cuanto a la salud mental y emocional. Para ello se aplicó el STAI y el PERMA. Como resultados, los futbolistas investigados muestran niveles promedio de ansiedad rasgo y buen nivel de bienestar general. También se encuentra correlación entre ambos. Este estudio permite concluir que si bien la situación actual puede verse como amenazante, mantener las condiciones laborales y en actividad para un deportista parece ser clave para sentir cier to grado de control frente a la incertidumbre, así como estrategias de afrontamiento que permitan tener una alta sensación de bienestar.

Palabras clave: Emociones, Coronavirus, Pandemia, Futbolista profesional.

Abstract. In response to the emergence of COVID 19 and the resulting pandemic, countries took steps to manage infection curves. They have forced them to remain in confinement and this strategy affected various contexts, within which is the sports field, suspending competitions, official matches, training on the field, sports broadcasts, etc. However, in the case of soccer professionals, they remained in physical preparation through technological platforms. The objective of this work is to explore the levels of trait anxiety, feeling of well-being and the relationship between both variables in professional soccer players from four clubs in Chile in order to contribute to the diagnosis of how the Covid-19 crisis may have affected sports reality in terms of mental and emotional health. For this, the STAI and PERMA were applied. As results, the investigated soccer players show average levels of trait anxiety and a good level of general well-being. There is also a correlation between the two. This study allows us to conclude that although the current situation can be seen as threatening, maintaining working and active conditions for an athlete seems to be key to feeling a certain degree of control in the face of uncertainty, as well as coping strategies that allow having a high sensation of wellness.

Key words: Emotions, Coronavirus, Pandemic, Professional soccer player.

\section{Introducción}

La actual condición mundial en relación con el brote de coronavirus, ha afectado a todos los ámbitos de la vida. Es una crisis de salud pública sin precedentes que se propagó rápidamente en el mes de diciembre del 2019 y que obligó a un aislamiento masivo de la población mundial, lo cual trajo consigo enormes costos sociales, económicos (Balluerka et al. 2020; Johnson et al., 2020; Bonaccorsi et al., 2020) y cambios en los hábitos saludables y estilos de vida (Santos-Miranda et al.,

Fecha recepción: 23-09-21. Fecha de aceptación: 21-01-22

Paula Francisca Ortiz Marholz

portizmarholz@yahoo.com
2022; Intelangelo et al., 2022). Dentro de ello, el ámbito deportivo no es la excepción, puesto que a nivel global se han visto perjudicadas competiciones de todos los niveles, espectáculos y acontecimientos deportivos, la industria del fitness, el turismo activo, entrenamientos suspendidos, la educación física escolar, actividades extraescolares, partidos oficiales de fútbol, etc. A nivel particular esta crisis ha afectado a los servicios deportivos y a todos los agentes involucrados: practicantes deportivos de todo nivel, empresarios, profesionales y técnicos asociados y a las organizaciones deportivas (Gambau, 2020).

Esta crisis obligó a muchos países a imponer estados de cuarentena y confinamiento en que la libertad de movimiento estaba restringida y con ello todas las prác- 
ticas deportivas formativas, de alto rendimiento, profesionales y recreativas. Particularmente en Chile, la emergencia sanitaria desatada por el COVID-19 propició que se desplegaran una serie de medidas económicas, sanitarias y de orden público. De esta manera, desde el 18 de marzo de 2020 el país comenzó un confinamiento que permitió mitigar la creciente curva de contagio (Benítez et al., 2020). Esto afectó directamente a la programación de los diversos campeonatos organizados por la Asociación Nacional de Fútbol Profesional (ANFP), suspendiéndose intempestivamente el 17 de marzo del mismo año. Posteriormente, esto generó que todos los planteles profesionales y sus cuerpos técnicos dispusieran medidas de aislamiento en sus hogares, transformándose - en la mayoría de los casos - las prácticas habituales de entrenamiento en sesiones virtuales.

El fútbol es un deporte de interacción, donde la especificidad debe estar en relación permanente entre los componentes o fundamentos psico-cognitivos, táctico-técnicos, físicos y coordinativos en correlación permanente con el modelo de juego adoptado y sus respectivos principios que le dan cuerpo (Tamarit, 2007). Es un deporte de habilidades abiertas de colaboración-oposición e invasión, por lo que entrenar con otros es parte esencial de su significado y hacerlo de manera aislada del contexto del juego, no es coherente y además alejado de las exigencias de la competencia real (MartínBarrero y Martínez-Cabrera, 2019). Para mantener la capacidad de resistencia y de fuerza y reducir el impacto negativo del período de confinamiento, se han dispuesto distintas estrategias tanto de entrenamiento como de recuperación (Domínguez et al., 2020; Christensen et al., 2011). Sin embargo, un período inusual sin entrenamiento o competencia también podría presentar un importante efecto sobre los estados emocionales o mentales de los jugadores (Jukic et al., 2020).

Es sabido que la práctica deportiva, en sus distintas fases de desarrollo, trae consigo múltiples dificultades (Tamminen y Holt, 2010). Dependiendo de estas fases, los deportistas se enfrentan a numerosas demandas. Algunas de ellas son transversales a la mayoría de los deportes (por ejemplo, soportar la presión ejercida por las personas significativas). En cambio, otras son específicas del tipo de deporte practicado (e.g., gestionar la atención focalizada ante una salida de tacos en atletismo, afrontar el miedo al error frente a una exhibición, etc.). Las características que componen los deportes se pueden concebir como el conjunto de conductas, habilidades y actitudes necesarias para un rendimiento exitoso (Cerin et al., 2000).
En este sentido, la ansiedad competitiva se ha revelado como una de las emociones de mayor interés (Núñez y García- Mas, 2017). Se podría decir que ello es debido a las consecuencias negativas que esta emoción puede conllevar si no es correctamente afrontada, tales como el sobreentrenamiento (Madigan et al., 2018) o una menor intención de continuar con la práctica deportiva (Pons et al., 2018) o emociones y miedos producidos por un confinamiento prolongado (Brooks et al., 2020).

Dentro del contexto deportivo, la ansiedad rasgo competitiva se define como la tendencia de los y las deportistas a significar como amenazantes las situaciones de evaluación, es decir, las competitivas, donde se observarían sentimientos de apresión y tensión ante ellas (Martens, 1977). Grossbard et al. (2009), plantean que la expresión de la ansiedad competitiva podría incluir manifestaciones somáticas, preocupación acerca del propio rendimiento y pérdida de concentración.

Un metaanálisis reciente realizado por Rocha y Osório (2018), muestra que los sujetos que practican deportes individuales tienden a mostrar mayores niveles de ansiedad competitiva que las personas que practican deportes colectivos. A su vez, distinguiendo los deportes según el tipo de habilidad perceptivo-motora entre habilidades abiertas o cerradas (clasificación propuesta por Knapp en 1963), se puede decir que un deporte de habilidades abiertas, trae consigo un amplio abanico de situaciones posibles (por no decir infinitas) durante la competición, así como la presencia de otros agentes implicados (contrincante). Ante esta situación, se desconoce de antemano la secuencia de ejecuciones que sucederán, siendo más probable que los y las deportistas se enfrenten a situaciones que no han anticipado. En esta clasificación se encuentran deportes como el tenis, el fútbol, el baloncesto, el balonmano, etc. Esta diferenciación entre entornos más o menos predecibles, podría generar diferencias en cuanto a la experiencia emocional de los y las deportistas (Ramis et al., 2013).

Ramis et al. (2013) pudo mostrar en su investigación, mayores niveles de ansiedad somática en los deportes de habilidad abierta y mayores niveles de preocupación en los deportes de habiliades cerradas. A su vez, valores similares de desconcentración en ambos grupos. Un estudio de Pons et al. (2020) mostró mayores niveles de ansiedad rasgo somática (i.e., tensión muscular) en deportes de habilidad cerrada, mientras que los deportes de habilidad abierta mostraron mayores niveles de ansiedad cognitiva (i.e., preocupación y desconcentración).

La ansiedad-estado se conceptualiza como un estado 
emocional transitorio que se caracteriza por sentimientos subjetivos de tensión y aprensión. Son percibidos conscientemente y se asocia a una mayor actividad del sistema nervioso autónomo (Spielberger et al. 1970). La ansiedad-rasgo se refiere a diferencias individuales relativamente estables en la propensión a la ansiedad, es decir, diferencias entre las personas en la tendencia a responder a situaciones percibidas como amenazantes con elevaciones en la intensidad de la ansiedad-estado (Spielberger et al., 1970). Serían las diferencias individuales en la semejanza de que una persona experimentaría ansiedad-estado en una situación estresante.

Otro aspecto psicológico importante es la sensación de bienestar, que es un concepto subjetivo, que tiene que ver más con la sensación de sentirse a gusto que con el disfrute de las condiciones objetivas que en principio producen este efecto. Se puede estar más o menos bien, aunque las condiciones externas no sean las mismas. Por ello, la medición del bienestar, si bien se basa en ciertas garantías materiales relacionadas con la satisfacción de necesidades vitales básicas (tales como la preservación de la vida, la integridad física, una alimentación suficiente y adecuada, el reguardo del cuerpo frente a las inclemencias externas mediante habitación y vestimenta apropiada y la salud) depende sobre todo de la actitud que se asume frente a la cobertura de las expectativas que se consideran individual y socialmente indispensables (Hernández et al., 2018). Ello explica que las perspectivas de bienestar sean distintas en distintas culturas y geografías, e incluso entre personas de una misma colectividad. El bienestar también es considerado como una medida intangible y no económica del éxito. No es solo ausencia de enfermedad, sino que se extiende al deseo de que un individuo «florezca»; se comprometa en la vida, tenga buenas relaciones con los demás, experimente emociones positivas y negativas y sea resistente a la adversidad (Diener y Seligman, 2004; Huppert, 2014, Ryan et al., 2019).

En la actualidad es evidente un creciente enfoque en el bienestar por parte de las comunidades científicas, políticas y comerciales, por ejemplo, se estudia regularmente en investigaciones cientificas (Windle et al., 2010; Boehm, Kubzansky, 2012), también se ha informado el desarrollo de nuevos tratamientos psicoterapéuticos para la depresión (Zilcha-Mano et al., 2014) y se ha utilizado como base para programas de promoción de la salud basados en el trabajo y la comunidad (Carolan et al., 2017).

El bienestar, pues, no es una categoría medible objetivamente, sino que está asociada a perspectivas cul- turales e individuales (Hernández et al. 2018). Los niveles de satisfacción hacia la vida, se mide sobre la base de estándares mínimos (cuyo conjunto definen la calidad de vida) pero también juega un papel importante el valor subjetivo que se les otorga (Satorres Pons, 2013). Ahora bien, es un hecho que el nivel de satisfacción vital de los individuos influye en su percepción de la realidad y en su propio funcionamiento vital, es decir, existe una evaluación mental subjetiva del bienestar (Hernández, 2017).

Una persona dotada de la capacidad de superación de la adversidad y de relativización de los aspectos negativos por otorgamiento de valor a los positivos posee un potencial mayor de felicidad que otra que se concentra en lo negativo (Hernández et al., 2018). En este sentido, Seligman (2011) propone un modelo multidimensional para la identificación de los niveles de bienestar y satisfacción con la vida enmarcados dentro del cumplimiento de cinco componentes: Emociones positivas, Compromiso, Relaciones positivas, significado y logro o autorrealización.

Todos estos aspectos, en su conjunto proporcionan el bienestar. Estas variables pueden ser medidas de forma subjetiva, ya que están definidas por lo que las personas piensan y sienten. Importante destacar que las relaciones personales positivas y los logros, poseen componentes subjetivos y objetivos (Selignan, 2014)

Por esta razón, conocer los niveles de ansiedad y bienestar puede entregar información crucial para generar estrategias de trabajo que impliquen mantener estados emocionales y percepciones de bienestar aún en circunstancias difíciles. En concreto, y dada la situación mundial que ha provocado en muchos países la aplicación de medidas sanitarias y de confinamiento, lo que a su vez ha generado síntomas físicos y psicológicos como estrés, ansiedad, trastornos del estado del ánimo y la percepción de bienestar general (Liu et al., 2020). El objetivo de esta investigación es identificar el estado de ansiedad rasgo (propensión de ver las situaciones como amenazantes) y la sensación de bienestar (actitud con la cual se enfrentan las situaciones) en jugadores de fútbol profesional confinados producto de la crisis COVID-19 y a su vez, determinar la posible relación entre ambas variables.

\section{Metodología}

\section{Diseño de investigación}

El estudio se caracteriza por ser una investigación de naturaleza cuantitativa, definiéndose como un estu- 
dio de carácter no experimental, de tipo descriptivo correlacional transeccional.

Este artículo se propone tres objetivos específicos:

1) Establecer los niveles de ansiedad-estado presentes en una muestra de futbolistas profesionales de clubes de la Primera División A del sistema de campeonatos de ANFP durante la emergencia sanitaria por COVID-19.

2) Determinar los niveles de bienestar presentes en una muestra de futbolistas profesionales de clubes de la Primera División A del sistema de campeonatos de ANFP durante la emergencia sanitaria por COVID-19.

3) Correlacionar niveles de ansiedad-estado y bienestar, en una muestra de futbolistas profesionales de clubes de la Primera División A del sistema de campeonatos de ANFP durante la emergencia sanitaria por COVID-19.

\section{Muestra}

En esta investigación se utilizó un muestreo no probabilístico de carácter intencionado, dada la variabilidad de la población y la posibilidad del equipo investigador de acceder fácilmente a algunos casos característicos (Otzen y Manterola, 2017). De esta manera, como se observa en la Tabla 1, participaron en esta investigación 93 futbolistas profesionales pertenecientes a cuatro clubes de la Primera División A, de los cuales dos pertenecen a la zona norte y dos a la Región Metropolitana-Santiago de Chile y que participan del sistema de campeonatos de la Asociación Nacional de Fútbol Profesional (ANFP). Conviene indicar que, la edad mínima de los participantes fue de 18 años y la máxima de 40 años, con una media de $24,7 \pm 5,6$. Del total de participantes, 24 jugadores tenían edades entre 18 y 20 años $(25,8 \%), 31$ entre 21 y 25 años (33,3\%), 18 entre 26 y 30 años (19,4\%), 17 entre 31 y 35 años $(18,3 \%)$ y tres entre 36 y 40 años $(3,2 \%)$.

\begin{tabular}{lcc}
$\begin{array}{l}\text { Tabla } 1 \\
\text { Participantes }\end{array}$ & & \\
\hline Club & N & $\%$ \\
\hline Club 1 & 32 & 34,4 \\
Club 2 & 11 & 11,8 \\
Club 3 & 31 & 33,3 \\
Club 4 & 19 & 20,4 \\
\hline
\end{tabular}

\section{Instrumentos}

Para la evaluación de los niveles de ansiedad, se utilizó el Inventario de Ansiedad estado-rasgo, STAI (Spielberger, et al, 1970). Es un instrumento autoinformado ampliamente utilizado para evaluar el rasgo de ansiedad y el estado de ansiedad en la población general, clínica (Beutel et al., 2011; Ronquillo, 2012) y también en el ámbito del deporte (Horikawa y
Yagi, 2012) .

El STAI consiste en una medida de informe, con dos escalas de 20 ítems cada una, que evalúa los niveles de ansiedad de estado y rasgo. De esta manera, STAI separa la intensidad de la experiencia transitoria en una situación específica (o un marco de tiempo limitado) de las diferencias individuales en la semejanza de experimentar ansiedad en diferentes situaciones, ambas desarrolladas como medidas unidimensionales. En la subescala ansiedad-estado, indican cuánto refleja cada declaración cómo se sienten ahora, en este momento. En la subescala de ansiedad-rasgo, los encuestados indican cómo se sienten generalmente.

La adaptación española de esta escala (Bermúdez, 1978a, 1978b), los valores de consistencia interna para muestras normales y clínicas son similares a los del estudio original, oscilando entre 0,82 y 0,92 . Para Chile, un estudio de Vera-Villarroel et al., (2007), obtuvo consistencia interna de .92 , para STAI-E y .87 para STAIR. Utilizado en el contexto deportivo presenta un coeficiente de fiabilidad alpha de Cronbach de .930 para los ítems que miden ansiedad/estado y .900 para los ítems ansiedad/rasgo (Johnson e Ivarsson, 2011; Spielberg et al., 1983). Otro estudio en deportistas muestra $\alpha=0.92$ para ansiedad/estado y á $=0.89$ para ansiedad/rasgo (Castro-Sánchez et al., 2018). Un estudio en futbolistas mostró $\mathrm{a}=.710 \mathrm{y} \mathrm{a}=.723$ (Zurita-Ortega et al., 2017).

En el presente estudio se utilizó la subescala de ansiedad-rasgo por ser aquella más estudiada en el ámbito deportivo y aquella sobre la cual se puede trabajar en caso de ser necesario.

Consecutivamente, el bienestar fue evaluado a través del instrumento PERMA de Butler y Kern (2016) que mide los cinco pilares del bienestar definidos por Seligman (2011): Emociones positivas, Compromiso, Relaciones positivas, significado y logro o autorrealización. Además, evalúa tres factores independientes: salud, emociones negativas y soledad. Esta escala es el producto de varios estudios desarrollados con una amplia muestra internacional: Malasia, Australia, Ecuador, entre otros (Khaw y Kern 2015; Kern et al., 2015; Lima et al., 2017). Tales estudios comprueban que la escala tiene un modelo con ajuste adecuado de cinco factores. La consistencia interna es aceptable para cada una de las cinco subescalas, pues reportan valores de validez predictiva: $\mathrm{P}=0.88 ; \mathrm{E}=0.72 ; \mathrm{R}=0.82 ; \mathrm{M}=$ $0.90 ; \mathrm{A}=0.79$, Escala total $=0.94$. En Chile los índices de consistencia de la escala total y de la mayoría de las dimensiones se ubicaron entre $\mathrm{a}=.65$ a .91 (Cobo- 
Rendón, Pérez, Díaz, 2019).

Con respecto al presente estudio, se analizará el factor criterial global, reconocido por evaluar la sensación de bienestar general y permite tener una aproximación general a la medida del bienestar (Hone et al., 2014).

Todos los jugadores firmaron un consentimiento informado para participar en la presente investigación.

\section{Análisis de datos}

Se utilizó el programa estadístico SPSS para Windows, versión 25.0. Se aplicó estadística descriptiva con medias, desviaciones estándar y percentiles. La prueba de normalidad de Kolmogorov-Smirnov (KS) entregó valores $\mathrm{p}<0,05$ razón por la cual se procedió a utilizar estadística no paramétrica, con pruebas de Kruskal-Wallis para comparar la ansiedad/rasgo y las dimensiones del PERMA según clubes y correlaciones de Spearman para relacionar los puntajes de ansiedad/ rasgo con cada dimensión del PERMA.

\section{Resultados}

Con respecto a la variable ansiedad-rasgo, los jugadores de todos los clubes $(1,2,3,4)$ se encuentran cercanos al percentil 50, situándose en el promedio de la población de mostrar una tendencia hacia conductas ansiosas, producto de su estructura de personalidad (ver tabla 2).

Tabla 2.

Comparación de los valores ansiedad/rasgo de la muestra según club. \begin{tabular}{lllllc}
\hline & $1(\mathrm{n}=32)$ & $2(\mathrm{n}=11)$ & $3(\mathrm{n}=31)$ & $4(\mathrm{n}=19)$ & $\mathrm{p}$ \\
\hline Ansiedad/rasgo & $20,4 \pm 5,4$ & $20,9 \pm 5,4$ & $19,5 \pm 9,3$ & $20,7 \pm 6,6$ & 0,896 \\
\hline
\end{tabular}

Con respecto a la sensación de bienestar, para Club 1 , se puede decir que el equipo tiene una alta sensación de bienestar global, encontrándose todos los índices por sobre el promedio. En el caso de las emociones positivas se encuentran en el promedio o normal funcionamiento, sin embargo, podemos hacer notar que es la variable más baja. En relación con Club 2, sus jugadores se encuentran con una alta sensación de bienestar. Se puede decir que los participantes del estudio tienen sensaciones de bienestar global que le permite suponer una buena actitud y valoración de su estado actual. Con respecto a Club 3, también muestra un alto funcionamiento con respecto a la sensación de bienestar global, siendo más alto el de salud, lo que podría ser un factor protector para los tiempos de COVID 19. Club 4 muestra el más alto nivel de funcionamiento con respecto a la sensación de bienestar global. A su vez, el compromiso pareciera ser la variable que mejor consideran dentro de la evaluación subjetiva (Tabla 3).
Tabla 3.

\begin{tabular}{cccccc}
\multicolumn{7}{c}{ Comparación de los valores de PERMA según club. } \\
\hline \multicolumn{7}{c}{$1(\mathrm{n}=32)$} & $2(\mathrm{n}=11)$ & $3(\mathrm{n}=31)$ & $4(\mathrm{n}=19)$ & $\mathrm{P}$ \\
\hline Emociones positivas & $7,5 \pm 1,9$ & $8,6 \pm 0,6$ & $8,3 \pm 2,3$ & $9,1 \pm 0,9$ & $0,002 * *$ \\
Compromiso & $8,4 \pm 1,9$ & $9,0 \pm 0,6$ & $8,8 \pm 1,7$ & $9,3 \pm 1,0$ & 0,062 \\
Relaciones positivas & $8,2 \pm 2,0$ & $9,0 \pm 0,7$ & $8,5 \pm 2,2$ & $9,1 \pm 0,8$ & 0,223 \\
Significado & $8,5 \pm 1,9$ & $9,3 \pm 0,5$ & $8,9 \pm 2,4$ & $9,2 \pm 1,0$ & $0,028^{*}$ \\
Logro & $7,6 \pm 1,9$ & $8,3 \pm 0,4$ & $8,3 \pm 1,3$ & $8,3 \pm 0,7$ & 0,268 \\
Global & $8,1 \pm 1,7$ & $8,8 \pm 0,4$ & $8,6 \pm 1,8$ & $9,0 \pm 0,7$ & $0,007 * *$ \\
$\mathrm{~N}$ & $3,4 \pm 2,3$ & $3,5 \pm 1,4$ & $2,8 \pm 2,9$ & $2,9 \pm 2,3$ & 0,355 \\
Salud & $8,6 \pm 1,9$ & $9,2 \pm 0,7$ & $9,4 \pm 0,8$ & $8,9 \pm 0,7$ & 0,128 \\
\hline
\end{tabular}

Finalmente, de acuerdo con la tabla 4, en el análisis global, se pudo determinar que existe una relación estadísticamente significativa entre ansiedad/rasgo y la variable de bienestar global. Por lo tanto, se podría decir que presentar niveles promedio de ansiedad/rasgo, se vincula con altos niveles de bienestar global.

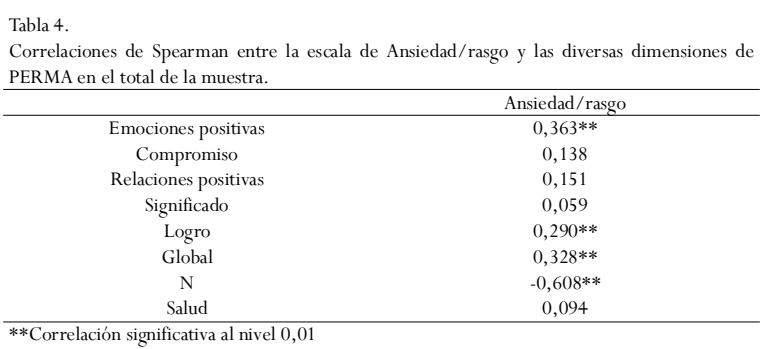

\section{Discusión}

En relación a los objetivos de este estudio, en primer lugar, se identificaron los niveles de ansiedad y de bienestar en jugadores de fútbol profesional confinados producto de la crisis COVID-19 y, en segundo lugar, se determinó la posible relación entre ambas variables.

Con respecto al primer objetivo, los futbolistas profesionales de los clubes estudiados muestran presencia de ansiedad-rasgo dentro del promedio de la población. La ansiedad rasgo se entiende como una disposición, tendencia o rasgo a diferencia de un estado emocional inmediato (ansiedad estado) caracterizado por sentimientos de tensión, aprensión y nerviosismo. La ansiedad rasgo debe ser inferida por la frecuencia que un individuo experimenta aumentos de sus estados de ansiedad, es así que personas con un alto nivel perciben un mayor rango de situaciones como amenazantes y están más propensos a mostrar conductas ansiosas (ansiedad estado) en frecuencia e intensidad (Ries et al., 2012).

En la situación mundial actual producto del COVID19 , se esperaría que la población y los deportistas muestren cambios en sus hábitos saludables (Intelangelo et al. 2022) y por ende se podría suponer que deportistas con un alto nivel de ansiedad rasgo muestren conductas, sentimientos y pensamientos caracterizados por la tensión, preocupación, molestia, negatividad, etc. (AcebesSánchez et al., 2022). En este estudio se muestra que los futbolistas de los clubes investigados, al encontrarse 
en el promedio de la población en cuanto a su ansiedad rasgo, han podido adaptarse a las situaciones cambiantes, concordando parcialmente con los hallazgos de Leguizamo et al. (2021) que encontraron que los niveles de ansiedad, estrés y síntomas depresivos de los atletas estudiados son relativamente bajos, sin embargo en el caso de los futbolistas estudiados, al presentar niveles de ansiedad rasgo promedio, se puede inferir una tendencia a ocupar estrategias de manejo de las emociones y pensamientos negativos, así como el caso del estudio de Leguizamo et al. (2021) donde mostró que el uso de estrategias de afrontamiento como la reestructuración cognitiva y la calma emocional se asoció con niveles más bajos de estados emocionales negativos. A su vez, podría asociarse a que el confinamiento no varió la percepción de competencia motriz tal como lo plantena los hallazgos de Cárcamo-Oyarzún et al. (2022). Y esto no genera aumento de la ansiedad.

En relación con segundo objetivo y segunda variable estudiada, el estado de bienestar, se encontró que la población estudiada muestra un alto nivel de bienestar global, lo que no es concordante con los hallazgos de Zhang y Ma (2020) quienes proponen que en esta crisis sanitaria se debe presentar especial atención a las personas que dejaron de trabajar durante el aislamiento y aquellas que eran físicamente activas y tuvieron de disminuir o suspender sus actividades, ya que podría verse más afectada su condición de bienestar general. En este sentido, puede ser que a pesar de que los futbolistas estudiados cambiaron su sistema de entrenamiento y competición, los clubes se encargaron que tuvieran los implementos necesarios, así como entrenamientos vía plataformas que los mantuvieran trabajando. En los deportistas el bienestar subjetivo es muy relevante pues contribuye a mejorar variables psicológicas asociadas a la práctica como motivación, autoconcepto y sensación de autoeficacia frente al desempeño deportivo. A su vez, el deportista acostumbrado fisiológicamente a mantener ciertas cargas de entrenamiento puede presentar cambios en el estado de ánimo, lo que tiene implicancias en el desempeño, por cambios fisiológicos y por cambios conductuales producto de las situaciones de estrés, encontrándose relación entre los estados de ánimo, las emociones y el bienestar con las posibilidades de lesionarse (Olmedilla et al., 2014).

En relación con el tercer objetivo de esta investigación, se encontró relación significativa entre ansiedad rasgo y bienestar. Un nivel de ansiedad promedio, se asocia a evaluar situaciones potencialmente amenazantes con la percepción de su controlabilidad y por conse- cuencia con la posibilidad de movilizar estrategias para su manejo lo que se vincularía con altos niveles de bienestar subjetivo. Lo que se asocia a los hallazgos de González et al. (2017) donde plantea el control del estrés previene alcanzar estados de ansiedad.Y también que el bienestar subjetivo como concepto global, puede ser clave a la hora de afrontar los problemas derivados del confinamiento por COVID-19, prevenir enfermedades mentales y gestionar las emociones, según lo planteado por Véliz et al. (2020).

Se presenta claramente entonces, la importancia de mantener niveles adecuados de entrenamientos en deportistas, en especial los profesionales, ya que además de los positivos efectos fisiológicos y cognitivos, la práctica deportiva potencia el bienestar psicológico, tal como lo plantea Pearson et al. (2014).

\section{Aplicaciones Prácticas}

A través del desarrollo de esta investigación, se ha podido entender y constatar la relevancia del bienestar subjetivo y cómo se puede relacionar con la tendencia a presentar conductas ansiosas, las cuales, si se encuentran en niveles promedio, podrían eventualmente actuar como factores protectores y potenciadores del bienestar. Así mismo creemos que el hecho de que los clubes y sus entrenadores muestren preocupación por la persona, por el cuidado por la salud física y mental, han contribuido a que los niveles de ansiedad se enmarquen en la sensación de lo controlable, a pesar de encontarnos en una situación mundial de incertidumbre por la presencia del COVID-19. Esto gracias a las medidas de confinamiento, con estrategias de entrenamiento en el hogar, mantención de condiciones laborales, entre otras acciones que han tenido como finalidad el mantener el estado físico y psicológico para el momento en que vuelvan a las competencias deportivas.

Se plantea también como importante la evaluación y el trabajo en el manejo de los niveles de ansiedad para generar mayores y mejores niveles de bienestar en los jugadores, lo que podría reflejarse en buenos rendimientos deportivos. Incorporando estrategias que disminuyan el estrés y la ansiedad por la vuelta a la competición con público, respetando los miedos y los tiempos propios de cada individuo, lo que también podría reflejarse en la prevención de lesiones y frustraciones.

\section{Referencias}

Acebes-Sánchez, J., Granado-Peinado, M. y Marchena, C. (2021). 
Relación entre inteligencia emocional y ansiedad en un club de fútbol sala de Madrid. Retos, 39, 643-648

Balluerka, N., Gómez, J., Hidalgo, M.D., Gorostiaga, A., Espada. J.P., Padilla, J.L. y Santed, M.A. (2020). Las Consecuencias Psicológicas de la COVID-19 y el Confinamiento. Informe de Investigación. Servicio de publicaciones de la Universidad del País Vasco, Euskal Herriko Unibertsitateko Argitalpen Zerbitzua.

Bermúdez, J. (1978a). Anxiety and performance. Revista de Psicología General y Aplicada, 151, 183-207.

Bermúdez, J. (1978b). Functional analysis of anxiety. Revista de Psicología General y Aplicada, 153, 617-634.

Beutel, ME.; Bleichner, F.;Von Heymann, F.; Tritt, K. y Hardt, J. (2011). Inpatient psychosomatic treatment of anxiety disorders: Comorbidities, predictors and outcomes. Int. J. Clin Health Psychol., 11, 443-457.

Boehm JK. y Kubzansky, LD. (2012). The heart's content: the association between positive psuchological well-being and cardiovascular health. Psychological bulletin, 138(4), 655. Doi: 10.1037/a0027448

Bonaccorsi, G.;Pierri,F.; Cinelli,M.; Flori,A.; Galeazri,A.; Porcelli, F.; Schmidt,A.L.;Valensise, C.M.; Scala,A.; Quattrociocchi,W. y Pammollo, F. (2020). Proceeding of the National Academy of Sciences of the United States of America. PNAS 117 (27), 15530-15535. https://doi.org/10.1073/ pnas. 2007658117

Brooks, S.K., Webster R.K, Smith L.E., Woodland L., Wessely S., Greenberg N. y Rubin G.J. (2020) The psychological impact of quarantine and how to reduce it: rapid review of the evidence, Lancet North Am. Ed.

Butler, J., y Kern, M. L. (2016). The PERMA-Profiler: A brief multidimensional measure of flourishing. International Journal ofWellbeing, 6(3), 1-48. doi:10.5502/ijw.v6i3.526

Cárcamo-Oyarzún, J., Romero-Rojas, F. y Estevan, I. (2022). Impacto de la pandemia por COVID-19 en la percepción de competencia motriz de escolares de la ciudad de Temuco, Chile. Retos, 43, 361-369.

Carolan, S., Harris, PR. y Cavanagh, K. (2017). Improving employee well-being and effectiveness: systematic review and metaanalysis of web-based psychological interventions delivered in the workplace. Journal of medical internet research, 19(7), e271. Doi: $10.2196 /$ jmir.7583

Castro-Sánchez, M.; Zurita-Ortega, F.; Chacón-Cuberos, R.; López-Gutiérrez, C.J. y Zafra-Santos, E. (2018). Emotional Intelligence, Motivational Climate and Levels of Anxiety in Athletes from Different Categories of Sports: Analysis through Structural Equations. Int.J. Environ. Res. Public Health, 15, 894. https: / / doi.org/10.3390/ijerph15050894

Cerin, E., Szabo, A., Hunt, N. y Williams, C. (2000). Temporal patterning of competitive emotions: A critical review. Journal of Sports Sciences, 18, 605-626. Doi:10.1080/ 02640410050082314

Christensen, P. M., Krustrup, P., Gunnarsson, T. P., Kiilerich, K., Nybo, L., y Bangsbo, J. (2011).VO2 kinetics and performance in soccer players after intense training and inactivity. Medicine and science in sports and exercise, 43(9), 1716-1724. https: / / doi.org/10.1249/MSS.0b013e318211c01a

Diener, E y Seligman, M.E. (2004). Beyond Money: Toward an economy of well-being. Psychological Science in public interest, 5(1), 1-31. Doi: 10.1111/j.0963-7214.2004.00501001 doi: $10.3390 /$ sports8040056

Domínguez,E. ,Arjol, J., Crespo R. y Fernández C. (2020). Regreso al entrenamiento y la competición en el fútbol profesional después de la alerta sanitaria del covid-19 con énfasis en los efectos del confinamiento durante el desentrenamiento. Revista de Preparación Física en el Fútbol. ISSN: 18895050.

Gambau, V. (2020). COVID-19: La crisis ha afectado a todos. Revista Española de Educación Física y Deportes- REEFD, 429, 15-18

González, G., Valdivia-Moral, P., Cachón, J., Zurita-Ortega, F. y Romero-Ramos, O. (2017). Influencia del control del estrés en el rendimiento deportivo: La autoconfianza, la ansiedad y la concentración en deportistas. Retos, 32, 3-6

Grossbard, J. R., Smith, R.E, Smoll, F.L.y Cumming, S.P. (2009). Competitive anxiety in young athletes: Differentiating somatic anxiety, worry and concentration disruption. Anxiety, Stress \& Coping, 22, 153-166. Doi: 10.1080/10615800802020643

Hernández, C., Hernández,V.y Prada, R. (2018). Adaptación del perfil PERMA de bienestar subjetivo para adultos mayores institucionalizados colombianos. Revista Ciencia y Cuidado, 15 (1), 83-97. Doi: 10.22463/17949831.1235

Hernández, V. (2017). Evaluación del bienestar subjetivo entre adultos mayores institucionalizados en Cúcuta, Norte de Santander (Tesis fin de máster). Universidad Internacional de la Rioja, España.

Hone, L.; Jarden,A; Schofield, G. y Duncan, S. (2014). Measuring flourishing: The impact of operational definitions on the prevalence of high levels of wellbeing. International Journal of Wellbeing, 4(1), 62-90. doi:10.5502/ijw.v4i1.4

Horikawa, M.yYagi,A.(2012).The relationshipsamong trait anxiety, state anxiety and the goal performance of penalty shoot-out by university soccer players. PLoS ONE, 7, e35727.

Huppert, F.A. (2014). The state of wellbeing science: Concepts, measures, interventions and policies. Wellbeing: A complete reference guide, 1-49

Iglesias, R. (1982). La Ansiedad Estado/Rasgo (STAI) en un grupo clínico (Síndrome tóxico). Memoria de Licenciatura, Facultad de Psicología, Universidad de Salamanca

Johnson, M.C., Saletti-Cuesta, L. yTumas , N. (2020). Emociones, preocupaciones y reflexiones frente a la paldemia del COVID19 en Argentina. Ciência \& Saúde Coletiva 25(1), 2447-2456. https://doi.org/ $10.1590 / 1413$ 81232020256.1.10472020

Itelangelo, L., Molina, N., Bevacqua, N., Mendoza, C., Guzmán,I. y Jerez-Mayorga, D. (2022). Effect of Confinement by COVID-19 on the lifestyle of the Univerity Population of Argentina: Evaluation of Psysical Activity, Food and Sleep. Retos, 34, 274-282.

Jukic, I., Calleja-González, J., Cos F., Cuzzolin F., Olmo J., Terrados N., Njaradi, N., 
Kern M, Waters L, Adler A y White, M. (2015). A multifaceted approach to measuring well-being in students: Application of the PERMA framework. The Journal of Positive Psychology; 10(3): 262-271.

Khaw D. y Kern M. (2015). A cross-cultural comparison of the PERMA model of well-being. Undergraduate Journal of Psychology at Berkeley, 8, 10-23.

Knapp, B. (1963). Skill in sports: The attainment of proficiency. London: Rutledge\& Keagan

Lima-Castro S., Peña Contreras E, Cedillo Quizphe C. y Cabrera VélezM.(2017).Adaptación del perfil PERMA en una muestra ecuatoriana. Eureka:Asunción (Paraguay) 14(1), 69-83

Madigan, D.J., Hill, A. P. y Mallinson-Howard, S.H. (2018). Perfectionism and training distress in junior athletes: The mediating role of coping tendencies. European Journal of Sport Sciences, 18, 713-721. Doi:10.1080/ 17461391.2018.1457082

Martens, R. (1977). Sport Competition anxiety test. Champaign: Human Kinetics

Martín-Barrero,A.y Martínez-Cabrera, F.I.(2019). El modelo de juego en el fútbol. De la concepción teórica al diseño práctico. Retos 36 543-551. https://doi.org/10.47197/ retos.v36i36.71021

Muñiz, J. y Fernández-Hermida JR. (2010). La opinión de los psicólogos españoles sobre el uso de los test. Papeles del Psicólogo, 31, 108-121.

Núñez,A. y García-Mas,A. (2017). Relación entre rendimiento y la ansiedad en el deporte: Una revisión sistemática. Retos. Nuevas tendencias en Educación Física, Deportes y Recreación, 32, 172 177.

Otzen, T.Y Manterola, C. (2017).Técnicas de Muestreo sobre una Población a Estudio. International Journal of Morphology (online), 35 (1), 227-232.

http:/ /dx.doi.org/10.4067/S0717-95022017000100037.

Pons, J., Ramis, Y., Viladrich, C. y Checa, I. (2020). Niveles de ansiedad y estilos de afrontamiento en función de las características perceptivo-motoras del deporte. Revista de Psicología del Deporte, 29 (2), 105-115

Pons, J., Viladrich, C., Ramis, Y. y Polman, R.C.J. (2018). The mediating role of coping between competitive anxiety and sport commitment in adolescents athletes. Spanish Journal of Psychology, 21, e7, 1-9. Doi: 10.1017/sjp.2018.8

Ramis,Y.,Torregrosa, M. y Cruz, J. (2013). Revisitando a Simon \& Martens: La ansiedad competitiva en deportes de iniciación. Revista de Psicología del Deporte, 22,77-83.

Rocha, V.V.S. y Osório F. de L. (2018) Associations between competitive anxiety, athlete characteristics and sport context: Evidence from a systematic review and meta-analysis. Revista de Psiquiatría Clínica, 45(3),67-74. Doi: 10.1590/010160830000000160

Ronquillo, H. (2012). Asociación entre condiciones oclusales, niveles de ansiedad rasgo- estado y desordenes temporomandibulares en un grupo de estudiantes de odontología. Carta Odontol., 1, 27-42

Ryan, J., Curtis, R., Olds,T., Edney, S., Vandelanotte, C., Plotnikoff,
R. y Maher, C. (2019). Psychometric properties of the PERMA profiler for measuring wellbeing in AustralianAdults. PLoS ONE, 14(12): e0225932. Doi: 10.1371/journal pone. 0225932

Santos-Miranda, E., Rico-Díaz, J., Carballo-Fazanes,A.y AbelairasGómez, C. (2022). Cambios en hábitos saludables relacionados con actividad física y sedentarismo durante un confinamiento nacional por covid-19. Retos, 43, 415-421.

Sassi R., Requena B., Milanovic L., Krakan. I., Chatzichristos, K. y Alcaraz, P. (2020). Strategies and Solutions for Team Sports Athletes in Isolation due to COVID-19. Sports (Basel, Switzerland) 8 (4), 56. https://doi.org/10.3390/sports 8040056

Satorres Pons, E. (2013). Bienestar psicológico en la vejez y su relación con la capacidad funcional y la satisfacción vital. Tesis doctoral inédita.Valencia: Universidad deValencia.

Seligman, M. (2011). Flourish: A visionary new understanding of happiness and well-being. Atria Books. ISBN: 9781439190760.

Seligman, M. (2014). Florecer: La nueva psicología positiva y la búsqueda del bienestar. México: Océano.

Spielberger, C, Gorsuch, R. y Lushene, R. (1970). Manual for the State-TraitAnxiety Inventory. PaloAlto, California, Consulting Psychologist Press. (Adapt. Española, TEA, 1982).

Spielberger, C.D.; Gorsuch, R. y Luschene, R. The State-Trait Anxiety Inventory; Psychologist Press: Palo Alto, CA, USA, 1970; ISBN 8809400933

Tamminen, K.A. y Holt, N.L. (2010). A meta-study of qualitative research examining stressor appraisals and coping among adolescents in sport. Journal of Sport Sciences, 28, 1563-1580. Doi:02640414.2010.512642

Tamarit, X. (2007). ¿Qué es la periodización táctica? Vivenciar el juego para condicionar el juego. Madrid, España:Editorial MC Sports.

Tarragona, M. (2013). Psicología Positiva yTerapias Constructivas: Una propuesta integradora. Terapia Psicológica, 1(31), 115-125.

Vera-Villarroel, P., Celis-Atenas, K., Córdova-Rubio, N., BuelaCasal, G. y Spielberger, C. (2007). Preliminary Analysis and Normative Data of the State-TraitAnxiety Inventory (STAI) in Adolescent and Adults of Santiago, Chile. Terapia psicológica, 25(2), 155-162.https://dx.d21oi.org/10.4067/S071848082007000200006

Windle, G., Hughes, D., Linck, P., Russell, I. yWoods, B. (2010). Is exercise effective in promoting mental well-being in older age? A systematic Review. Aging \& mental health, 14(6), 652659

Zilcha-Mano, S., Dinger, U., McCarthy, KS., Barrett, MS.y Barber, JP. (2014). Changes in well-being and quality of life in a randomized trial comparing dynamic psychotherapy and pharmacotherapy for major depressive disorder. Journal of affective disorders, 152, 538-542. Doi: 10.1016/ j.jad2013.10.015

Zurita-Ortega, F.; Rodríguez-Fernández,S.; Olmo-Extremera,M.; Castro-Sánchez, M.; Chacón-Cuberos, R. y Cepero-González, M. (2017).Análisis de la resiliencia, ansiedad y lesión deportiva en fútbol según nivel competitivo. Cultura Ciencia y Deporte 12 (35), 135-142. ISSN 1696-5043 